Original Article

\title{
DEVELOPMENT AND VALIDATION OF SEVEN PHENYL HYDRAZINE CHLORO ESTER ISOMERS (PGIS) BY RP-HPLC-UV METHOD IN ANTICOAGULANT DRUG SUBSTANCE; APIXABAN
}

\author{
SARADHI VENKATA RAMANA V.a, ${ }^{*}$, DURGA RAJA K.a,b, RAGHU BABU K.a, PADMA M.a, JAGADEESH KUMAR V.b, \\ PAVAN KUMAR K. S. R. ${ }^{b}$, HEMANT KUMAR SHARMA
}

aDepartment of Engineering Chemistry, Andhra University, Vishakhapatnam, Andhra Pradesh, India 53003, bAnalyical Research

Department, Aurobindo Pharma Limited Research Centre-II, Indrakaran (V), Kandi (M), Sangareddy Dist, Telangana, India 502329 Email: saradhivundavilli@gmail.com

Received: 28 Nov 2019, Revised and Accepted: 22 Jan 2020

\begin{abstract}
Objective: The objective of this work was to develop and validate a simple and sensitive reverse-phase high-pressure liquid chromatography method for the determination of seven potential genotoxic impurities in Apixaban drug substance.

Methods: The optimized separation was achieved by using ACE 3 C18 PFP (150 mm×4.6 mm, $3 \mu \mathrm{m})$ HPLC column. The mobile phase-A was a degassed mixture of $0.01 \mathrm{M}$ Ammonium acetate buffer( $\mathrm{P}^{\mathrm{H}}$ adjusted $4.9 \pm 0.05$ with diluted glacial acetic acid) and mobile phase- $\mathrm{B}$ was a degassed mixture of Acetonitrile, Isopropyl alcohol and Buffer $\mathrm{P}^{\mathrm{H}} 4.9$ in the ratio of 60:20:20 v/v/v. The gradient program was operated at a flow rate of 1.0 $\mathrm{ml} / \mathrm{min}$ and $\mathrm{UV}$ detection was at $330 \mathrm{~nm}$.

Results: The method was superior at linearity for seven impurities and correlation coefficient values were larger than 0.999, moreover, in the separation point of view, this method further achieved no matrix interference through chromatography by better resolution of the other impurities from the Apixaban drug substance and its related impurities for the accurate analysis of seven potential genotoxic impurities. The established limits of detection (LOD), limits of quantification (LOQ) values for the seven mutagenic impurities were each of $5 \mathrm{ppm}(0.015 \mu \mathrm{g} / \mathrm{ml}) \mathrm{and} 15 \mathrm{ppm}$ $(0.045 \mu \mathrm{g} / \mathrm{ml})$ respectively. The developed method was validated as per ICH guidelines and applied as a generic method to determine these seven potential genotoxic impurities for the pharmaceutical process control and drug material release.
\end{abstract}

Conclusion: Validation of this analytical method was carried out including stability, selectivity, linearity, accuracy, system precision, method precision and intermediate precision thus proving that the described RP-HPLC method could be employed for fast and simple analysis of sevenphenyl hydrazine chloro ester isomers in Apixaban drug substance.

Keywords: Seven potential genotoxic impurities, Reverse phase-HPLC-UV, Method development, Validation, Apixaban drug substance

(C) 2020 The Authors. Published by Innovare Academic Sciences Pvt Ltd. This is an open access article under the CC BY license (http://creativecommons.org/licenses/by/4.0/] DOI: http://dx.doi.org/10.22159/ijcpr.2020v12i2.37507. Journal homepage: https://innovareacademics.in/journals/index.php/ijcpr

\section{INTRODUCTION}

Apixaban is chemically described as '1-(4-methoxyphenyl)-7-oxo-6[4-(2-oxopiperidin-1-yl) phenyl]-4, 5, 6, 7-tetrahydro-1H-pyrazolo [3,4c] pyridine-3-carboxamide', molecular formula is $\mathrm{C}_{25} \mathrm{H}_{25} \mathrm{~N}_{5} \mathrm{O}_{4}$ and molecular weight is 459.5. Apixaban is an anticoagulant and is routinely used in the treatment of stroke, embolism, infarct etc. Blood clotting profile in such patients needs to be monitored frequently. Apixaban has an oral bioavailability of $\sim 50 \%$. It is administered as a twice-daily dose. It is excreted in urine and feces. Apixaban is useful in atrial fibrillation, venous thromboembolism and pulmonary embolism [1]. Based on efficacy studies, it is confirmed that apixaban and rivaroxaban drugs were equally effective anticoagulation therapies that exhibited similar preventive effects against postoperative VTE after lumbar spine surgery [2]. Apixaban has the advantage of reduced bleeding compared with rivaroxaban, it was recommended as the first choice anticoagulant after lumbar spine surgery. As per prescribed information detailed in ELIQUIS [3]. Apixaban is formulated as oral tablets and marketed by Bristol-Myers Squibb Company with the trade name ELIQUIS. According to Less-Than-Lifetime (LTL) exposures phenomena of ICH M7 [4] for mutagenic impurities in the pharmaceutical industry, the risk assessment for carryover into the drug substance from the raw materials and intermediates to be appropriately controlled to fulfill the regulatory requirements. To achieve this, genotoxic assessment (identification of genotoxic impurity, control of impurity and generated data presentation) is must w. r. $t$ analytical approach and control of identified impurities based on TTC concept.

According to TTC concept, we have developed a new RP-HPLC method for quantification of seven identified potential genotoxic impurities in Apixaban drug substance, as these impurities having hydrazone and alkyl halide structural alerts. Hydrazines and hydrazones show conventional structural alerts for genotoxicpotentiality [5]. Alerting structural elements are mainly based on the nature of the electrophilic character (as such or based on its metabolic activation) and/or genotoxicity data from representative compounds. Evaluation of drug substances for potential genotoxicity encompasses effects on both genes and chromosomes. Whereas, for impurities, testing is focused on the relation of the potential interaction with DNA. The chemical reactivity that makes them useful in chemical synthesis brings about the risk that, a number of such molecules may react with DNA, leading to carcinogenesis. A molecule bearing an alkyl halide moiety is normally flagged by the most commonly used silico systems like Quantitative Structure-Activity Relationships (QSARs), StructureActivity Relationships (SARs) and consequently, an Ames assay test is carried out. The selected seven impurities shows mutagenic activity based on these two alerts. Hence genotoxicity is assumed, staged and further TTC concept is applied [4]. According to the maximum daily dose (5 $\mathrm{mg}$ twice/day), the allowed limit is not more than $150 \mu \mathrm{g} / \mathrm{g}$ for each impurity. Based on this reverse phase HPLC method developed and validated for the same limit. PGI-B(Ethyl 2chloro-2-[(4-methoxyphenyl)hydrazono] acetate) is used as a key raw material in the preparation of Apixaban. Where, this raw material is coupled with Apixaban intermediate-I and gives Apixaban intermediate-II, further in subsequent stages Apixaban is formed. Scheme has shown in fig. 1 and other PG impurities PGI-A, PGI-C, PGI-D, PGI-E, PGI-F and PGI-G are likely possible from PGI-B and structures of these impurities are given in same scheme. There have been many reported methods used to detect and quantify hydrazine and hydrazone related impurities [6-10] and alkyl halides 
[11-16] by using different analytical techniques. The main achievement of the present research was to develop an analytical method based on reverse phase HPLC for the separation and determination of seven potential genotoxic impurities in Apixaban drug substance. During the method development, we have considered Apixaban related substances for method specificity and the details are presented in specificity experiments in the following sections. The newly developed method was validated as per ICH guidelines [17].

\section{MATERIALS AND METHODS}

\section{Standards, reagents and study samples}

All seven (PGIs) impurities were obtained from APL Research Centre-II, A division of Aurobindo pharma LTD., Hyderabad, India. Ammonium acetate (EMPARTA® ACS), Isopropyl alcohol (HPLC GRADE) were procured from Merck (Mumbai, India). Glacial Acetic acid was procured from Sigma-Aldrich fine chemicals (st Louis, MO). Milli-Q water was used with the help of Millipore Purification system (Millipore $®$, USA). All Study samples of Apixaban drug substances were obtained from the division of APL Research center-II, A division of Aurobindo pharma LTD., Hyderabad, India.

\section{Chromatographic conditions}

The analysis was carried out at column oven temperature $35^{\circ} \mathrm{C}$, by using a HPLC column ACE 3 C18 PFP(150 mm×4.6 mm,3 $\mu \mathrm{m})$. The Mobile phase-A consists of $0.01 \mathrm{M}$ Ammonium acetate buffer (adjusted to $\mathrm{pH} 4.9 \pm 0.05$ with dilute glacial acetic acid), filtered through $0.45 \mu$ or finer porosity membrane filter. Mobile phase-B was a degassed mixture of Acetonitrile, Isopropyl alcohol and Buffer (pH $4.9 \pm 0.05$ adjusted with glacial acetic acid) in the ratio of $60: 20: 20 \mathrm{v} / \mathrm{v}$. The optimized gradient program was $0.01 \mathrm{~min}-45 \% \mathrm{~B}$, 15 min-60\%B, 25 min- $80 \%$ B, 27 min- $45 \%$ B, 35 min- $45 \%$ B. The prepared mobile phase was delivered at a constant flow rate of 1.0 $\mathrm{ml} / \mathrm{min}$. The injection volume was $20 \mu \mathrm{l}$ and the impurity analytes were detected by UV at $330 \mathrm{~nm}$.

\section{Preparation of standard solutions}

Prepared a degassed mixture of Mobile phase-A and Mobile phase-Bin the ratio of 50:50 v/v and used as the diluent. A stock solution of seven (PGIs) impurities was prepared by sonicating to dissolve in the Mobile phase-B, as stock concentration of $300 \mu \mathrm{g} / \mathrm{ml}$. Dilute this solution to the concentration of $15 \mu \mathrm{g} / \mathrm{ml}$ with diluent. Final concentration of the above solution was further diluted to $0.45 \mu \mathrm{g} / \mathrm{ml}$ in the diluent (Standard solution). A test sample of Apixaban drug substance was prepared at a concentration of $3 \mathrm{mg} / \mathrm{ml}$ by sonicate to dissolving initially is in the mobile phase- $\mathrm{B}$, followed by mobile phase-A. The final standard solution was used as the System suitability solution and the resolution between Imp-C and Imp-D is not less than 1.5.

\section{Instrumentation and software for operation}

A waters e2695 separation module with 2998 PDA detector and UV detector with online degasser (made: Waters corporation, Milford, MA, USA), Shimadzu LC-2030Cwith UV detector (made: Shimadzu corporation, Japan.) and Shimadzu LC-2030 plus Prominence-iseries with UV detector (made: Shimadzu corporation, Japan.) were carried out for method development and validation. The Analytical precise balances GE 412, CPA225D and Ultra Microbalance-MSA2.75-000DM (made: Goettingen, Germany) were used for weighing the samples and impurities for sample and impurity standard solution preparations. EUTECH-PH $700 \mathrm{pH}$ meter was used for the adjustment of $\mathrm{pH}$ in the mobile phase. The chromatograms were recorded and analyzed through empower 3 software.

\section{Challenges in quantitative analysis}

The scenario for the chromatography is where the drug substance matrix components elute early, the separation of impurities and all other related substances is a big challenge to the development. The first step of method development was carried out with a standard solution of $15 \mu \mathrm{g} / \mathrm{ml}$ and with the columns Ascentis ${ }^{\circledR}$ Express F5 $(150 \mathrm{~mm} \times 4.6 \mathrm{~mm}, 2.7 \mu \mathrm{m})$ and Poroshell EC-C18 (4.6 $\mathrm{mm} \times 150 \mathrm{~mm}$, $2.7 \mu \mathrm{m})$. The MP-A: B(45:55), the temperature of the column kept $35^{\circ} \mathrm{C}$, the Flow rate was $0.7 \mathrm{ml} / \mathrm{min}$ was used to separate the seven impurities. The impurities were not enough to separate with each other and eluted early and not even separated from all other known related substances.

The column selectivity for the resolution of all other known related substance was hard enough, because of similar chemical structure and polarities. This was overcome with the help of ACE 3 C18 PFP $(150 \mathrm{~mm} \times 4.6 \mathrm{~mm}, 3 \mu \mathrm{m})$. Different mobile phases with different polarity compositions were used for their separation efficiency viz: water-Acetonitrile, water-methanol, water-Isopropyl alcohol, sodium phosphate buffers and phosphate buffers with methanol. All these were not given better separation with all known related substances and good peak shapes of analytes.

Eventually, Ammonium acetate solution $0.01 \mathrm{M}(\mathrm{pH} \quad 4.9 \pm 0.05$ adjusted with diluted glacial acetic acid) and Acetonitrile, Isopropyl alcohol and buffer mixtures (60:20:20 v/v/v) were selected with proper investigations as it yielded the best resolution between the all known related substances and seven mutagenic isomeric impurities with sample matrix background. The $\mathrm{pH}$ of the buffer plays a crucial role for separation of Imp-F and Imp-G, because they were adjacent and better separation was achieved with $\mathrm{pH} 4.9 \pm 0.05$.

\section{Method validation}

The optimized method was validated as per the ICH guidelines in terms of specificity or selectivity, the limit of quantification LOQ, limit of detection LOD, precision, linearity, and accuracy.

\section{Specificity (or) selectivity}

Specificity (or) Selectivity is the ability of the method to check the analyte response in the presence of all seven impurities (Imp-A, Imp-B, Imp-C, Imp-D, Imp-E, Imp-F and Imp-G) including all other process-related substances which were used in the synthetic process of Apixaban drug substance. For specificity determination, all known related substances were prepared and injected individually to confirm retention times. The solutions of diluent, Apixaban drug substance (control sample), Apixaban drug substance spiked with seven potential impurities (spiked sample)and Apixaban drug substance spiked seven potential impurities spiked sample with all known related substances(All spiked sample) were injected to confirm any co-elution with seven (PGI's) impurities peaks from any known related substances. The represented chromatograms of sample and spiked sample and spiked sample with all other known related substances were expressed in fig. 2.

Peak purity of all known analyte peaks met the acceptance criteria i.e. Purity angle (AG) should be less than purity threshold (TH). The results shown that there was no interference for analyte peaks. Represented in table 1

\section{Limit of detection (LOD) and limit of quantification (LOQ)}

Limit of detection (LOD) and Limit of Quantification (LOQ) for seven (PGI's) analytes peaks were determined, based on the response of analytes. The predicted concentrations of LOD, LOQ for seven analytes were prepared and injected each solution six times in to the HPLC by followed the developed method conditions. The précised LOD and LOQ values are presented in table 2 . The evaluated results indicated that this method was significantly sensitive to carry out the quantification of impurities in Apixaban drug substance at low ppm-level.

\section{Linearity}

A series of solutions were prepared by using seven analytes at concentration levels from LOQ to $150 \%$ of specification levels (LOQ $0.060 \mu \mathrm{g} / \mathrm{ml}, \quad 0.090 \mu \mathrm{g} / \mathrm{ml}, \quad 0.11 \mu \mathrm{g} / \mathrm{ml}, \quad 0.22 \mu \mathrm{g} / \mathrm{ml}, \quad 0.34 \mu \mathrm{g} / \mathrm{ml}$, $0.45 \mu \mathrm{g} / \mathrm{ml}, 0.56 \mu \mathrm{g} / \mathrm{ml}$ and $0.67 \mu \mathrm{g} / \mathrm{ml}$ ) and each solution was injected in to HPLC as per methodology. From this linear regression data for all the analytes tested is presented in table 2 .

\section{Accuracy}

The Accuracy of the proposed experimental method was done, using the standard addition technique. It was performed by spiking known amounts of all seven impurities at four different concentration levels ranging from $0.045 \mu \mathrm{g} / \mathrm{ml}$ (LOQ), $0.22 \mu \mathrm{g} / \mathrm{ml} \quad(50 \%), 0.45 \mu \mathrm{g} / \mathrm{ml}$ 
(100\%), and $0.67 \mu \mathrm{g} / \mathrm{ml}(150 \%)$ into Apixaban drug substance. It was carried out each in triplicate injections at these level concentrations. The obtained values of the test compound were within the specified limit of $80-120 \%$. The complete accuracy experimental results were shown in table 3 .

\section{Precision}

The precision experiment (system precision and method precision) of the developed method was studied by taking final standard solution $(0.45 \mu \mathrm{g} / \mathrm{ml})$ injected in six replicates for system precision and six different preparations of sample spiked with a final standard solution for method precision into the proposed method. The relative standard deviation values for the response area of all impurity analyte peaks were calculated and found to be less than $5.0 \%$. The concluded values of repeatability and reproducibility of the test method showed that this method is sufficiently precise. The experimental precision values are presented in table 3.

Table 1: Specificity (or) selectivity experimental data

\begin{tabular}{lllll}
\hline Name of the impurity & $\begin{array}{l}\text { RT } \\
\text { (min) }\end{array}$ & Peak purity of control sample & Peak purity of a spiked sample & Peak purity of All spiked sample \\
\cline { 2 - 5 } & 10.32 & 0.2410 .829 & Purity (AG)/Purity(TH) & Purity (AG)/Purity(TH) \\
\hline Imp-A & 13.79 & 0.1730 .901 & 0.2440 .688 & 0.2100 .897 \\
Imp-B & 14.73 & 0.2191 .123 & 0.1700 .740 & 0.1520 .952 \\
Imp-C & 15.24 & 0.3501 .205 & 0.3001 .043 & 0.2901 .167 \\
Imp-D & 17.60 & 0.2680 .943 & 0.3501 .097 & 0.2301 .219 \\
Imp-E & 20.82 & 0.2331 .048 & 0.7180 .926 & 0.3461 .012 \\
Imp-F & 21.20 & 0.2890 .937 & 0.4250 .823 & 0.1891 .077 \\
Imp-G & & & & 0.3131 .001 \\
\hline
\end{tabular}

Table 2: LOD, LOQ and linearity experimental data

\begin{tabular}{|c|c|c|c|c|c|c|c|}
\hline Parameter & Imp-A & Imp-B & Imp-C & Imp-D & Imp-E & Imp-F & Imp-G \\
\hline $\mathrm{LOD}(0.015 \mu \mathrm{g} / \mathrm{ml})(\% \mathrm{RSD}) \#$ & 3.2 & 3.1 & 3.0 & 2.1 & 4.3 & 4.8 & 2.9 \\
\hline $\mathrm{LOQ}(0.450 \mu \mathrm{g} / \mathrm{ml})(\% \mathrm{RSD}) \#$ & 0.7 & 0.5 & 0.6 & 0.6 & 0.9 & 1.9 & 1.2 \\
\hline \multicolumn{8}{|l|}{ Linearity } \\
\hline Slope & 83185 & 80139 & 99544 & 76630 & 74888 & 88478 & 82079 \\
\hline Intercept & 133 & 199 & 136 & 193 & 39 & 185 & 123 \\
\hline $\begin{array}{l}\text { Correlation coefficient } \\
\text { \#:six injections }\end{array}$ & 0.9999 & 0.9999 & 0.9999 & 0.9999 & 0.9999 & 0.9999 & 0.9999 \\
\hline
\end{tabular}

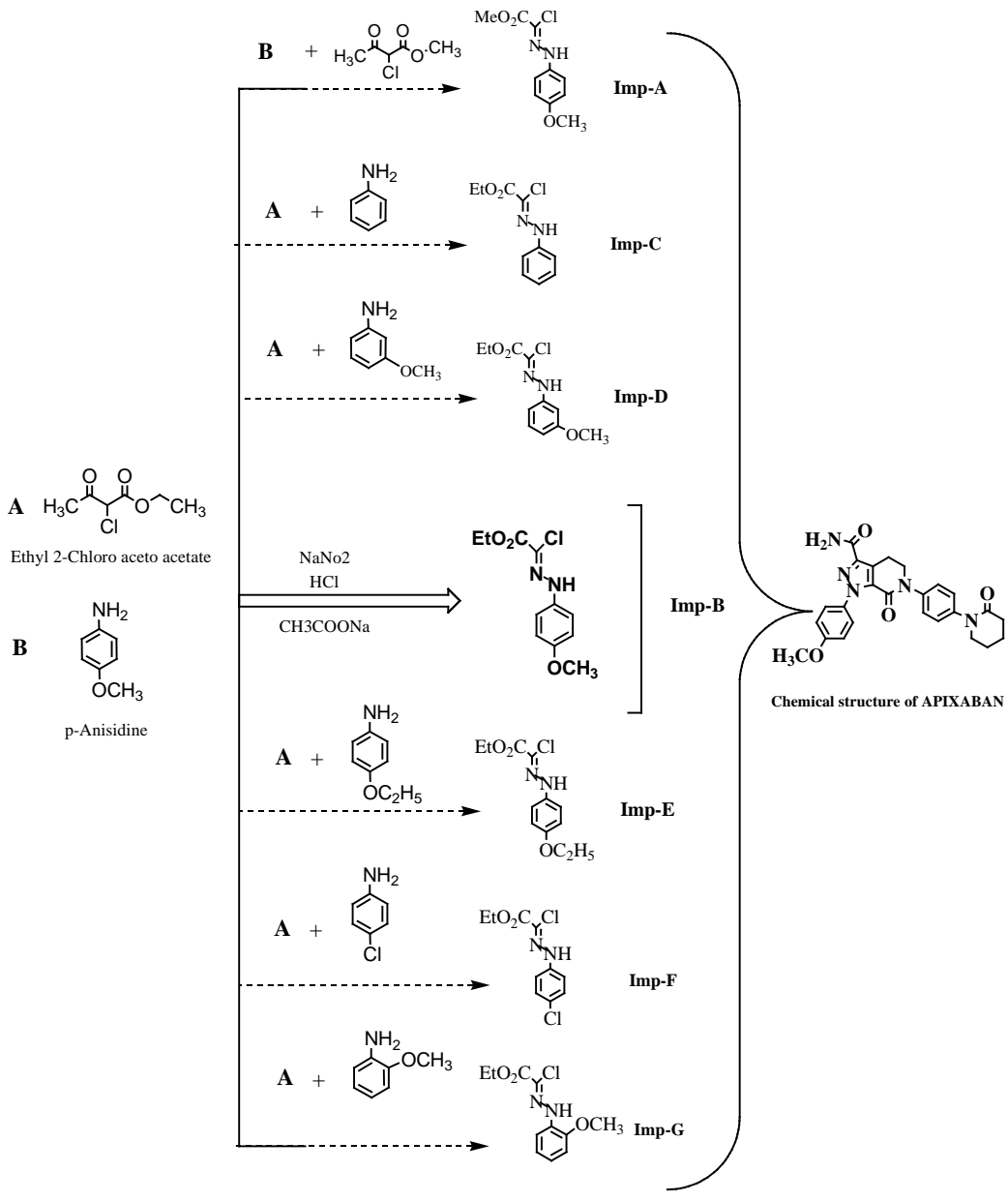


Fig. 1: Schematic representation for the formation of seven PGIs and Apixaban drug substance

Table 3: Precision (system precision and method precision) and accuracy experimental data

\begin{tabular}{|c|c|c|c|c|c|c|c|}
\hline Parameter & Imp-A & Imp-B & Imp-C & Imp-D & Imp-E & Imp-F & Imp-G \\
\hline Systemprecision* & $0.1 / 1.0$ & $0.4 / 0.7$ & $0.6 / 0.9$ & $0.6 / 1.2$ & $0.5 / 0.9$ & $0.3 / 1.4$ & $0.5 / 0.1$ \\
\hline Method precision \# & $94.1 / 1.5$ & $97.3 / 1.1$ & $97.3 / 1.1$ & $96.6 / 1.6$ & $96.6 / 1.1$ & $101.3 / 2.5$ & $105.2 / 2.1$ \\
\hline LOQ Accuracy^ & $100.0 / 1.8$ & $102.5 / 0.4$ & $102.2 / 1.0$ & $95.2 / 3.9$ & $96.2 / 0.8$ & $96.2 / 2.9$ & $93.8 / 0.4$ \\
\hline $50 \%$ Accuracy $^{\wedge}$ & $95.7 / 0.4$ & $100.3 / 0.6$ & $98.4 / 0.4$ & $97.0 / 0.9$ & $96.2 / 0.8$ & $91.3 / 1.0$ & $90.8 / 0.1$ \\
\hline $100 \%$ Accuracy $^{\wedge}$ & $94.8 / 0.6$ & $98.9 / 0.4$ & $98.0 / 0.5$ & $97.7 / 0.6$ & $95.6 / 0.4$ & $103.0 / 1.2$ & $108.1 / 0.5$ \\
\hline $150 \%$ Accuracy $^{\wedge}$ & $87.9 / 0.8$ & $93.5 / 0.9$ & $92.1 / 0.7$ & $90.2 / 0.7$ & $89.7 / 0.8$ & 89.6/1.1 & $103.6 / 0.8$ \\
\hline
\end{tabular}

*Intra/Inter day and six replicate injections experimental values, \#Six injections average experimental values/\% RSD, ^${ }^{\wedge}$ Three injections average experimental values/\% RSD

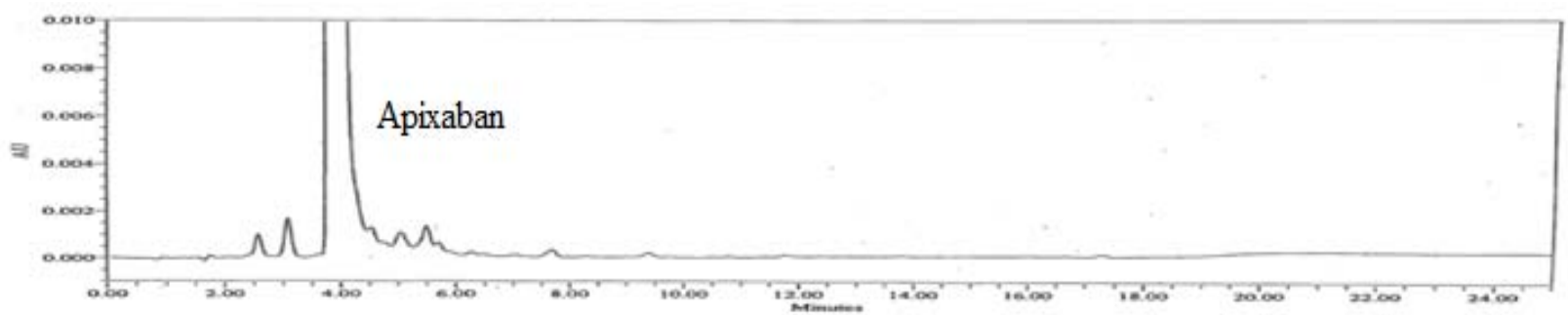

[A]

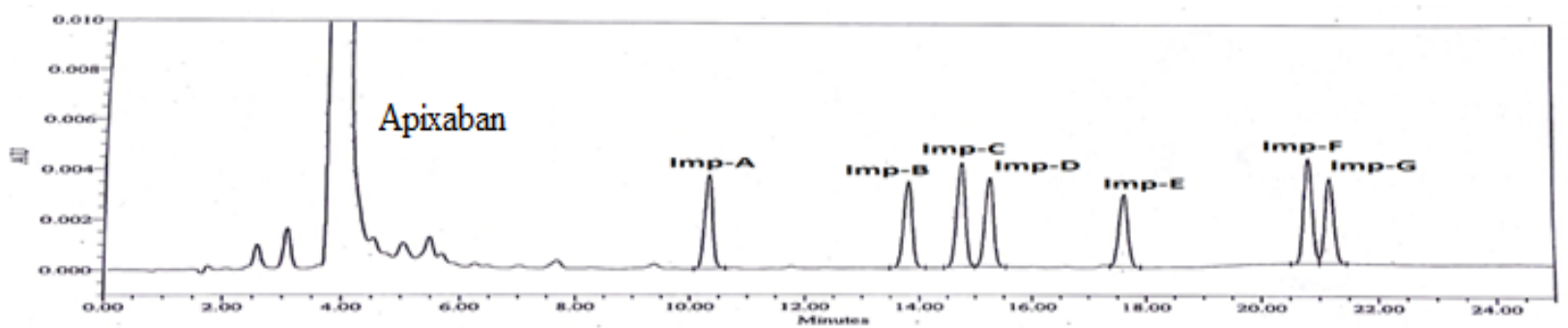

[B]

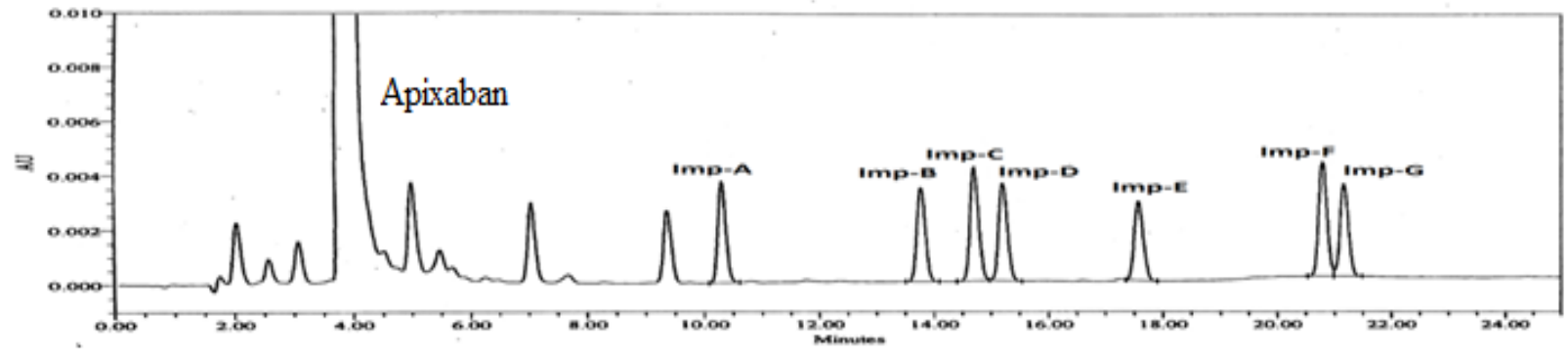

[C]

Fig. 2: [A] A typical representation chromatogram of Apixaban drug substance, [B] Chromatogram of Apixaban drug substance spiked with seven (PGIs) analytes, [C] Chromatogram of Apixaban drug substance spiked with seven (PGIs) analytes and all other process-related impurities

\section{CONCLUSION}

The main aspect for optimization of successful reverse phase-HPLC method for the quantification of seven potential genotoxic impurities in Apixaban drug substance as follows, the analytical method must be precise, selective, linear and accurate for daily analysis in quality control labs. This simple, reliable and rapid RPHPLC method with UV detection was developed for the separation and quantification of seven (PGIs) impurities in Apixaban drug substance. This method is validated as per ICH requirements, the results of specificity, precision (System precision and Method precision), linearity, Accuracy were in good agreement with the followed limits and used for the analysis of routine sample analysis of Apixaban drug substance.

\section{ABBREVIATION}

RP-HPLC-Reverse phase High-pressure liquid chromatography, TTCThreshold of toxicological concern, PGI-Potential genotoxic impurity, ICH-International conference of harmonization, LOD-Limit of detection and LOQ-Limit of quantitation.

\section{ACKNOWLEDGMENT}

The work was supported by the APL Research centre-II (A division of Aurobindo Pharma Limited, Hyderabad, India.) and we would like to acknowledge the Chemical research department, Analytical department for their support of this work.

\section{FUNDING}




\section{AUTHORS CONTRIBUTIONS}

All the author has contributed equally.

\section{CONFLICT OF INTERESTS}

\section{Declared none}

\section{REFERENCES}

1. Fazeel ZA. Apixaban: an oral anticoagulant having a unique mechanism of action with better safety and efficacy profile. MAMC J Med Sci 2016;2:63-8.

2. Zhang Kun, Zhao Shenghao, Kan Wusheng, Xiao Jun, Pu Feifei, $\mathrm{Li}$ Kun. Comparison of apixaban and rivaroxaban for anticoagulant effect after lumbar spine surgery: a single-center report. Future Sci 2018;4:297.

3. Eliquis, US FDA Label. Bristol-Myers Squibb Company; 2016.

4. International Conference on Harmonization of technical requirements for registration of pharmaceuticals for human use, M7, Assessment and control of DNA reactive (Mutagenic) impurities in pharmaceuticals to limit potential carcinogenic risk; 2014.

5. Sawatari K, Nakanishi Y, Matsushimi T. Relationships between chemical structures and mutagen city: a preliminary survey for a database of mutagen city test results of new workplace chemicals. Ind Health 2001;39:341-5.

6. Jenny Wang, Samuel Yang, Kelly Zhang. A simple and sensitive method to analyze genotoxic impurity hydrazine in pharmaceutical materials, a simple and sensitive method to analyze genotoxic impurity hydrazine in pharmaceutical materials. J Pharm Biomed Anal 2016;126:141-7.

7. Elder DP, Snodin D, Teasdale A. Control and analysis of hydrazine, hydrazides and hydrazones-genotoxic impurities in active pharmaceutical ingredients (APIs) and drug products. J Pharm Biomed Anal 2011;54:900-10.

8. Raman NVVSS, Prasad AVSS, Reddy KR. Sensitive derivatization methods for the determination of genotoxic impurities in drug substances using hyphenated techniques. J Pharm Biomed Anal 2014;89:276-81.
9. Khan M, Kumar S, Jayasree K, Krishna Reddy KVSR, Dubey PK. Simultaneous trace level determination of potentially genotoxic hydrazine, methyl hydrazine, and alkylamines in pharmaceutical substances by $\mathrm{CE}$ using indirect photometric detection. Chromatographia 2013;76:801-9.

10. Jagota NK, Chetram AJ, Nair JB. Determination of trace levels of hydrazine in the penultimate intermediate of a novel antiinfective agent. J Pharm Biomed Anal 1998;16:1083-7.

11. Gorog S, Balogh G, Cschi A. Estimation of impurity profile in drugs and related materials. Part-II-the role of chromatographic and spectroscopic methods in the estimation of side-reactions in drug syntheses. J Pharm Biomed Anal 1993;11:1219-26.

12. Huybrechts T. Successfully developing and validating methods for the quantification of genotoxic impurities in APIs. Informa Genotoxic Impurities Meeting, Hilton Prague; 2007.

13. Lee CB, Hubert M, Nguyen Van Dua C. Determination of N, NDimethylaminoethyl chloride and the dimethylaziridiniumionat sub-ppm levels in diltiazem hydrochloride by LC-MS with electrospray ionization. Analyst 2000;125:1255-9.

14. Hansen SH, Sheribah ZA. Comparison of CZE, MEKC, MEEKC and non-aqueous capillary electrophoresis for the determination of impurities in bromazepam. J Pharm Biomed Anal 2005;39:322-7.

15. Ellison GK. Development of a general headspace GC-ECD method for genotoxic alkyl halides at trace levels in API-the challenge of genotoxic impurities and their analysis. Pharmaceuticals Analysis science Group (PASG) Autumn Meeting, Milton Keynes UK; 2006.

16. Liu DQ, Sun M, Korda AS. Recent advances in trace analysis of pharmaceutical genotoxic impurities. J Pharm Biomed Anal 2010;51:999-1014.

17. International Conference on Harmonization of technical requirements for registration of pharmaceuticals for human use, Q2 (R1), Validation of Analytical Procedures: Text and Methodology; 2005. 